\title{
A Computational Method to Calculate the Exact Solution for Acoustic Scattering by Fluid Spheroids
}

\author{
J. D. Gonzalez, E. F. Lavia, S. Blanc \\ Underwater Sound Division. Argentinian Navy Research Office (DIIV) UNIDEF (National \\ Council of Scientific and Technical Research - Ministry of Defense). Laprida 555. (1638) Vicente \\ López.Buenos Aires. Argentina. silblanc@yahoo.com
}

July 3,2018

\begin{abstract}
The problem of scattering of harmonic plane acoustic waves by fluid spheroids (prolate and oblate) is addressed from an analytical approach. Mathematically, it consists in solving the Helmholtz equation in an unbounded domain with Sommerfeld radiation condition at infinity. The domain where propagation takes place is characterised by density and sound speed values $\rho_{0}$ and $c_{0}$, respectively, while $\rho_{1}$ and $c_{1}$ are the corresponding density and sound speed values of an immersed object that is responsible of the scattered field. Since Helmholtz equation is separable in prolate/oblate spheroidal coordinates, its exact solution for the scattered field can be expressed as an expansion on prolate/oblate spheroidal functions multiplied by coefficients whose values depend upon the boundary conditions verified at the medium-immersed fluid obstacle interface. The general case $\left(c_{0} \neq c_{1}\right)$ is cumbersome because it requires to solve successive matrix systems that are ill-conditioned when $c_{1} / c_{0}$ is far from unity. In this paper, a numerical implementation of the general exact solution that is valid for any range of eccentricity values and for $c_{0} \neq c_{1}$, is provided. The high level solver code has been written in the Julia programming language while a software package recently released in the literature has been used to compute the spheroidal functions. Several limit cases (Dirichlet and Neumann boundary conditions, spheroid tending to sphere) have been satisfactorily verified using the implemented code. The corresponding example scripts can be downloaded from the authors' web (GitHub) site. The numerical implementation of the exact solution leads to results that are in agreement with reported results obtained through approximate solutions for far-field and nearfield regimes. Additionally, the new code has been used to extend results reported in the literature.
\end{abstract}

\section{Introduction}

The interaction of harmonic plane sound waves with prolate/oblate spheroids has been widely and increasingly investigated, mainly for soft and rigid scatterers, along the last six decades in different branches of acoustics 1, 2, 3. In particular, underwater acoustics can be considered quite proliferous in reported articles on different aspects of this topic due to its relevance in applications to fisheries management and marine ecosystem research.

Many developed models provide exact or approximate solutions with harmonic time dependence for the general problem of acoustic scattering by spheroids which have been extensively applied to several aquatic organisms and objects immersed in the ocean [4, 5, 6]. 
Both for soft and rigid scatterers, computational implementations of approximate solutions have been published for either near or far-field conditions.

However, less attention has received the case of penetrable spheroidal scatterers till 1964 when the corresponding analytical solution for the oblate case was published for the first time [7]. Three years later numerically computed results of the scattered wave radiation patterns were reported only for a particular case of sound speed ratio, $c_{0} / c_{1}=1$, being $c_{0}$ and $c_{1}$ are the sound speed in the medium and the fluid prolate spheroid, respectively [8]. In 1988 Furusawa [4] numerically computed acoustic backscattering patterns from fluid prolate spheroids for specific cases when the sound speed and density ratios verify $c_{0} / c_{1} \approx 1$ and $\rho_{0} / \rho_{1} \approx 1$. Several authors have presented results in the last years [9, 10] valid for the same range of parameters. More recently, another reference [11] considers the general case $c_{0} \neq c_{1}$ and $\rho_{0} \neq \rho_{1}$ but only for spheroids with low eccentricity.

Mathematically, the problem of acoustic scattering of plane waves with harmonic dependence consists in solving Helmholtz equation in an unbounded domain with Sommerfeld radiation condition at infinity. The domain where propagation takes place is characterised by density and sound speed values $\rho_{0}$ and $c_{0}$, respectively, while $\rho_{1}$ and $c_{1}$ are the corresponding density and sound speed values of an object immersed in that medium that is responsible of the scattered field. Different boundary conditions must be verified for the medium-object interface according to the type of scatterer considered, namely, soft, rigid and penetrable or fluid objects (i.e. Dirichlet, Neumann and transmission boundary conditions, respectively). The solution of the scalar wave equation strongly depends on the object's shape and exact solutions only exist for a few cases. When the object is a sphere, the canonical case, the exact solution is based on an expansion in spherical wave functions [12. For scatterers of spheroidal shape, the exact solution can be expanded on prolate/oblate spheroidal wave functions as a result of applying separation of variables to Helmholtz equation expressed in spheroidal coordinates [13, 14].

In this paper a new computer implementation based on the exact solution for fluid spheroids valid for any value of eccentricity and arbitrary $c_{0}, c_{1}, \rho_{0}, \rho_{1}$, is provided. The implementation of this solution was developed using recently available computational codes by Adelman et al. [15, 16] (which will be called AGD software from now on) together with a high level layer code implemented in the Julia programming language [17].

As it is well known, for many cases of interest (e.g. $k a \gg 1$, being $k$ the wave number and $a$ a typical longitudinal dimension of the scatterer) computation of spheroidal wave functions requires precision beyond the one provided by hardware floating point numbers [18] (currently 64 bits in the consumer market). In fact, the AGD code implements floating point arbitrary precision through the use of a specialized $\mathrm{C}++$ library. The Julia language is a relatively new free programming language with some features that make it especially attractive for the implementation of the spheroidal wave function related code, that is the possibility of having floating point arbitrary precision arithmetic and algebra, both features built in right out of the box. On the other hand, its calculation speed compared to other high-level equivalent computational environments constitutes a very advantageous feature.

This paper is structured as follows. In Section 2 the analytical exact solution is rederived. Basically, it is a refurbished derivation that closely follows the previous work by Yeh [7] but contemplating both cases, prolate/oblate spheroids, and correcting some minor typos. This section provides the basis for subsequent formulation and establishes the nomenclature that is used in the whole article. Section 3 explains the algorithmic procedure of the coefficient's calculation. In Section 4 it is verified that results derived from the numerical implemented solution agree with well known limit cases, namely, the penetrable spheroid behaves like the impenetrable ones (Dirichlet and Neumann conditions) and from 
the geometrical viewpoint, when the spheroid tends to the sphere. Computations of nearfield are also included as well as a comparison with previous published results. Section 5 exposes results of interest for some applications in acoustical oceanography, mainly in fisherires acoustics. In the illustrated examples the numerical implementation allows for extending the applicability ranges of previously published results [6, 19].

In Section 6, the computational implementation of the numerical solution under the Julia idiosyncrasy is described as well as a brief explanation on the sintaxis of the presented computational code is given. Finally, the conclusions of the work are summarized in Section (7.

\section{Analytical exact solution}

When acoustic scattering by spheroids is considered, it is convenient to use a spheroidal curvilinear coordinate system $(\xi, \eta, \varphi)[13$. The relationship between the prolate spheroidal coordinates and the Cartesian coordinates, is given by the following transformation [15]:

$$
\left\{\begin{array}{c}
x=\frac{d}{2}\left[\left(\xi^{2}-1\right)\left(1-\eta^{2}\right)\right]^{1 / 2} \cos \varphi \\
y=\frac{d}{2}\left[\left(\xi^{2}-1\right)\left(1-\eta^{2}\right)\right]^{1 / 2} \sin \varphi \\
z=\frac{d}{2} \xi \eta
\end{array}\right.
$$

where $d$ is the interfocal distance of the ellipse of major semi-axis $a=(d / 2) \xi$ and minor semi-axis $b=(d / 2)\left(\xi^{2}-1\right)^{1 / 2}$ (See Figure 1). The values for the three prolate spherical coordinates must lie between the following bounds:

$$
1 \leq \xi, \quad-1 \leq \eta \leq 1, \quad 0 \leq \varphi<2 \pi
$$

The prolate spheroidal coordinate $\xi$-range of variation is directly associated with the shape of the spheroid, ranging from $\xi=1$ (corresponding to the segment of length $d$ between both spheroid foci) to $\xi$ tending to $\infty$ (corresponding to a sphere of radius $a=b$ ). The surface of the prolate spheroid coincides with the coordinate surface given by equation $\xi=\xi_{0}$, with $\xi_{0}=\left(1-(b / a)^{2}\right)^{-1 / 2}$ and $d=2\left(a^{2}-b^{2}\right)^{1 / 2}$.
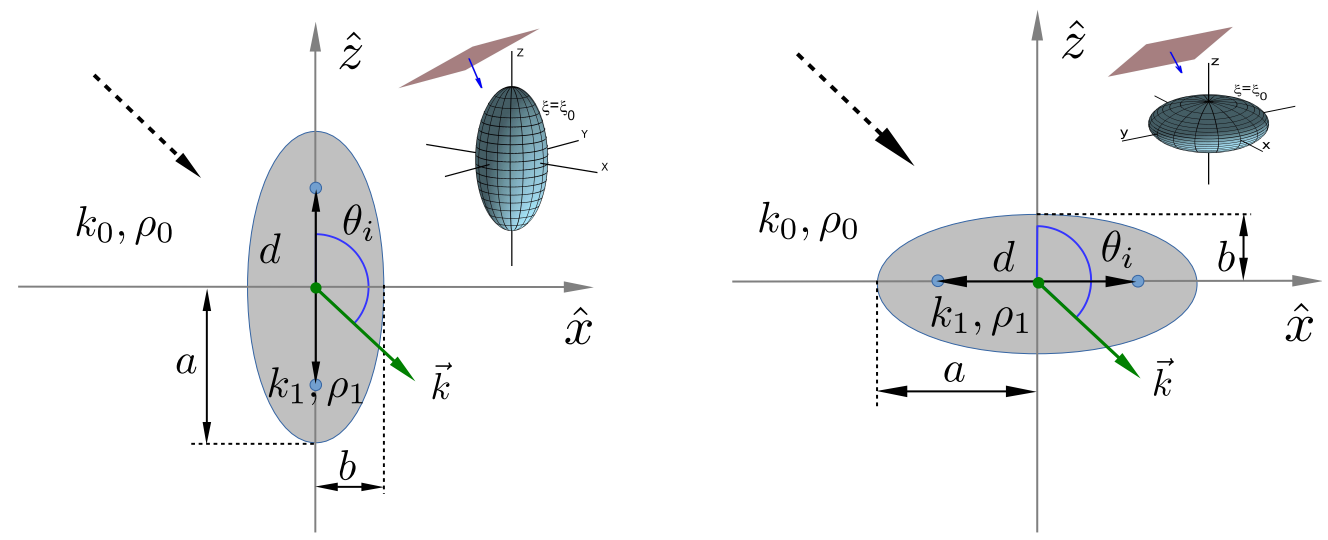

Figure 1: Prolate (left) and oblate (right) coordinate system and related conventions. 
On the other hand, the relationship for oblate spheroidal coordinates is given by

$$
\left\{\begin{array}{c}
x=\frac{d}{2}\left[\left(\xi^{2}+1\right)\left(1-\eta^{2}\right)\right]^{1 / 2} \cos \varphi \\
y=\frac{d}{2}\left[\left(\xi^{2}+1\right)\left(1-\eta^{2}\right)\right]^{1 / 2} \sin \varphi \\
z=\frac{d}{2} \xi \eta,
\end{array}\right.
$$

where coordinates ranges are

$$
\xi \geq 0, \quad-1 \leq \eta \leq 1, \quad 0 \leq \varphi<2 \pi
$$

and the surface of the oblate spheroid coincides with the coordinate surface given by equation $\xi=\xi_{0}$ where $\xi_{0}=\left((a / b)^{2}-1\right)^{-1 / 2}$.

For harmonic wave fields propagating in an unbounded medium, the acoustic pressure exterior and interior to a prolate (oblate) spheroidal surface of a penetrable scatterer immersed in that medium is governed by Helmholtz equation

$$
\nabla^{2} p+k^{2} p=0
$$

which is separable in prolate (oblate) spheroidal coordinates. Thus, the problem of solving the Helmholtz equation in an unbounded domain with the Sommerfeld radiation at infinity is reduced to solving two ordinary differential equations for radial and angular prolate (oblate) spheroidal functions, $R(h, \xi)$ and $S(h, \eta)$, respectively [1, 17, 15, 20], where $h \equiv$ $(d / 2) k$ is an adimensional parameter.

At this point is worth noting that the rule $\xi \rightarrow i \xi$ and $h \rightarrow-i h$ provides a direct transformation from the prolate to the oblate case [15, 20]. Therefore, from now on all mathematical expressions are valid for the fluid prolate spheroid and can be easily transformed to the oblate spheroid through the given conversion rule.

The acoustic pressure corresponding to an incident plane wave with angular frequency $\omega$ can be written as

$$
p_{i}=p_{0} \exp \left(i k_{0} \hat{k} \cdot \vec{r}\right),
$$

where $k_{0}=\omega / c_{0}$ is the wave number, $\hat{k}=\left(\sin \theta_{i} \cos \varphi_{i}, \sin \theta_{i} \sin \varphi_{i}, \cos \theta_{i}\right)$ is the incidence direction (being $\theta_{i}, \varphi_{i}$ the spherical angles of incidence) and the amplitude $p_{0}$, a real number.

Without loss of generality, due to the simmetry of revolution around the $z$ axis, we can consider $\varphi_{i}=0$ so that $\hat{k}=\left(\sin \theta_{i}, 0, \cos \theta_{i}\right)$. Such incident plane acoustic wave on a prolate/oblate spheroid is schematically illustrated in Figure 1, including both prolate and oblate coordinate systems used in this paper.

The incident pressure can be expanded on prolate spheroidal functions [21] so that at an arbitrary field point $\vec{r}=\vec{r}(\xi, \eta, \varphi)$,

$$
p_{i}(\vec{r})=2 p_{0} \sum_{\substack{m \geq 0 \\ n \geq m}} i^{n} \frac{\epsilon_{m}}{N_{m n}} S_{m n}\left(h_{0}, \cos \left(\theta_{i}\right)\right) S_{m n}\left(h_{0}, \eta\right) R_{m n}^{(1)}\left(h_{0}, \xi\right) \cos (m \varphi),
$$

where the subscripts $m, n$ are natural numbers, $S_{m n}$ are the angular spheroidal wave functions, $R_{m n}^{(1)}$ are the radial spheroidal wave functions of first kind, $i$ is the imaginary complex 
unit $\left(i^{2}=-1\right), \epsilon_{m}$ is the Neumann factor, defined as $\epsilon_{m}=2$ if $m \neq 0$ and $\epsilon_{m}=1$ if $m=0$ and $N_{m n}$ are the norms, given by

$$
N_{m n}=\int_{-1}^{1} S_{m n}^{2}\left(h_{0}, \eta\right) d \eta .
$$

The analytical solutions for the scattered and transmitted acoustic pressure, $p_{s}(\vec{r})$ and $p_{t}(\vec{r})$, respectively, can be expressed as,

$$
\begin{aligned}
& p_{s}(\vec{r})=2 p_{0} \sum_{\substack{m \geq 0 \\
n \geq m}} i^{n} A_{m n} \frac{\epsilon_{m}}{N_{m n}} S_{m n}\left(h_{0}, \cos \left(\theta_{i}\right)\right) S_{m n}\left(h_{0}, \eta\right) R_{m n}^{(3)}\left(h_{0}, \xi\right) \cos (m \varphi), \\
& p_{t}(\vec{r})=2 p_{0} \sum_{\substack{m \geq 0 \\
n \geq m}} i^{n} B_{m n} \frac{\epsilon_{m}}{N_{m n}} S_{m n}\left(h_{0}, \cos \left(\theta_{i}\right)\right) S_{m n}\left(h_{1}, \eta\right) R_{m n}^{(1)}\left(h_{1}, \xi\right) \cos (m \varphi) .
\end{aligned}
$$

The subscripts 0 and 1 (see Figure 1) refers to the surrounding medium and to the interior of the prolate spheroidal penetrable obstacle, respectively, and $A_{m n}$ and $B_{m n}$ are the unknown expansion coefficients. They are determined applying appropriate boundary conditions at the medium-immersed penetrable spheroid interface, that means to apply for the continuity of the pressure and the normal component of media particle velocity at the boundary, given by $\xi=\xi_{0}$. In symbols,

$$
\begin{gathered}
\left.\left(p_{i}+p_{s}\right)\right|_{\xi=\xi_{0}}=\left.p_{t}\right|_{\xi=\xi_{0}} \\
\left.\frac{1}{\rho_{0}} \frac{\partial\left(p_{i}+p_{s}\right)}{\partial \xi}\right|_{\xi=\xi_{0}}=\left.\frac{1}{\rho_{1}} \frac{\partial p_{t}}{\partial \xi}\right|_{\xi=\xi_{0}} .
\end{gathered}
$$

In order to compute the $A_{m n}$ and $B_{m n}$ coefficients, it is convenient to expand $S_{m n}\left(h_{0}, \eta\right)$ in terms of $\left\{S_{m \ell}\left(h_{1}, \eta\right)\right\}$

$$
S_{m n}\left(h_{0}, \eta\right)=\sum_{\ell=m}^{\infty} \alpha_{n \ell}^{(m)} S_{m \ell}\left(h_{1}, \eta\right),
$$

where

$$
\alpha_{n \ell}^{(m)}=\frac{\int_{-1}^{1} S_{m n}\left(h_{0}, \eta\right) S_{m \ell}\left(h_{1}, \eta\right) d \eta}{\int_{-1}^{1} S_{m \ell}^{2}\left(h_{1}, \eta\right) d \eta} .
$$

Substituting Equation (6) in the LHS of Equations (4) and (5) and using the orthogonality properties of the family $\left\{S_{m n}\left(h_{1}, \eta\right) \cos (m \varphi): m \geq 0, n \geq m\right\}$ lead to a matrix system involving the $A_{m n}$ and $B_{m n}$ coefficients.

Defining the $Q_{n \ell}^{(m)}$ and $f_{\ell}^{(m)}$ as

$$
\begin{gathered}
Q_{n \ell}^{(m)}=i^{n} \frac{1}{N_{m n}} \alpha_{n \ell}^{(m)} S_{m n}\left(h_{0}, \cos \left(\theta_{i}\right)\right)\left[\frac{\rho_{1}}{\rho_{0}} \frac{R_{m n}^{(3)^{\prime}}\left(h_{0}, \xi_{0}\right) R_{m \ell}^{(1)}\left(h_{1}, \xi_{0}\right)}{R_{m \ell}^{(1)^{\prime}}\left(h_{1}, \xi_{0}\right)} R_{m n}^{(3)}\left(h_{0}, \xi_{0}\right)\right], \\
f_{\ell}^{(m)}=-\sum_{n=0}^{\infty} i^{n} \frac{1}{N_{m n}} \alpha_{n \ell}^{(m)} S_{m n}\left(h_{0}, \cos \left(\theta_{i}\right)\right)\left[\frac{\rho_{1}}{\rho_{0}} \frac{R_{m n}^{(1)^{\prime}}\left(h_{0}, \xi_{0}\right) R_{m \ell}^{(1)}\left(h_{1}, \xi_{0}\right)}{R_{m \ell}^{(1)^{\prime}}\left(h_{1}, \xi_{0}\right)} R_{m n}^{(1)}\left(h_{0}, \xi_{0}\right)\right],
\end{gathered}
$$

where the $\xi$ derivative is indicated with a prime (i.e. ${ }^{\prime} \equiv d / d \xi$ ), it can be shown that the $A_{m n}$, with $(n=m, m+1, .$.$) , verify$

$$
\sum_{n=m}^{\infty} A_{n}^{(m)} Q_{n \ell}^{(m)}=f_{\ell}^{(m)} \quad \ell=m, m+1, \ldots
$$


where $m$ is indicated as superscript to emphasize the fact that for each fixed $m$, the left hand side of Equation 10 is a product of a row vector $\left(A_{m}^{(m)}, A_{m+1}^{(m)}, \ldots, A_{n}^{(m)}, \ldots\right)$ and a matrix $Q_{n \ell}^{(m)}$ while the right side is a row indexed by $\ell$.

After having computed the $A_{m n}$, it follows that the other coefficients $B_{m n}$, associated to the transmitted field, can also be calculated as

$$
B_{m \ell}=\sum_{n=m}^{\infty} i^{n-\ell} \frac{N_{m \ell}}{N_{m n}} \alpha_{n \ell}^{(m)} \frac{S_{m n}\left(h_{0}, \cos \left(\theta_{i}\right)\right)}{S_{m \ell}\left(h_{0}, \cos \left(\theta_{i}\right)\right)}\left[\frac{R_{m n}^{(1)}\left(h_{0}, \xi_{0}\right)+A_{m n} R_{m n}^{(3)}\left(h_{0}, \xi_{0}\right)}{R_{m \ell}^{(1)}\left(h_{1}, \xi_{0}\right)}\right] .
$$

In the far-field limit it can be shown [7] that

$$
p_{s}\left(r, \theta_{s}, \varphi_{s}\right) \approx p_{0} \frac{e^{i k_{0} r}}{r} f_{\infty}\left(\theta_{s}, \varphi_{s}\right)
$$

where $r, \theta_{s}, \varphi_{s}$ are the spherical polar coordinates of the observation point and $f_{\infty}\left(\theta_{s}, \varphi_{s}\right)$ is the so-called far-field scattering amplitude function which is widespreadly used in different acoustic scattering applications. For the particular case of our spheroid, it is

$$
f_{\infty}\left(\theta_{s}, \varphi_{s}\right)=\frac{2}{i k_{0}} \sum_{\substack{m \geq 0 \\ n \geq m}} A_{m n} \frac{\epsilon_{m}}{N_{m n}} S_{m n}\left(h_{0}, \cos \left(\theta_{i}\right)\right) S_{m n}\left(h_{0}, \cos \left(\theta_{s}\right)\right) \cos \left(m \varphi_{s}\right) .
$$

\section{Algorithmic procedure}

The numerical computation of the $A_{m n}$ and $B_{m n}$ coefficients starts with a truncation procedure. The series in Equation $(10)$ is truncated at $M$, so that for each value of $m$, there are $M-m+1$ unknown $A_{n}^{(m)}$, where index $n$ takes natural values in the interval $[m, M]$. Thus, $A_{n}^{(m)}$ satisfies the linear system

$$
\sum_{n=m}^{M} A_{n}^{(m)} Q_{n \ell}^{(m)}=f_{\ell}^{(m)} \quad \ell=m, m+1, . ., M
$$

where $A^{(m)}$ and $f^{(m)}$ are row arrays $\in \mathbb{C}^{1 \times(M-m+1)}$ and $Q^{(m)}$ is a matrix $\in \mathbb{C}^{(M-m+1) \times(M-m+1)}$.

The successive matrix systems obtained as a consequence of truncation, Equation (13), are

- for $m=0$,

$$
\left(A_{0}^{(0)}, A_{1}^{(0)}, . ., A_{M}^{(0)}\right)\left(\begin{array}{cccc}
Q_{0,0}^{(0)} & Q_{0,1}^{(0)} & \cdots & Q_{0, M}^{(0)} \\
Q_{1,0}^{(0)} & Q_{1,1}^{(0)} & \cdots & Q_{1, M}^{(0)} \\
\vdots & \vdots & \ddots & \vdots \\
Q_{M, 0}^{(0)} & Q_{M, 1}^{(0)} & \cdots & Q_{M, M}^{(0)}
\end{array}\right)=\left(f_{0}^{(0)}, f_{1}^{(0)}, . ., f_{M}^{(0)}\right)
$$

- for $m=1$,

$$
\left(A_{1}^{(1)}, A_{2}^{(1)}, . ., A_{M}^{(1)}\right)\left(\begin{array}{cccc}
Q_{1,1}^{(1)} & Q_{1,2}^{(1)} & \cdots & Q_{1, M}^{(1)} \\
Q_{2,1}^{(1)} & Q_{2,2}^{(1)} & \cdots & Q_{2, M}^{(1)} \\
\vdots & \vdots & \ddots & \vdots \\
Q_{M, 1}^{(1)} & Q_{M, 2}^{(1)} & \cdots & Q_{M, M}^{(1)}
\end{array}\right)=\left(f_{1}^{(1)}, f_{2}^{(1)}, . ., f_{M}^{(1)}\right)
$$


- ...

- for $m=M-1$,

$$
\left(A_{M-1}^{(M-1)}, A_{M}^{M-1}\right)\left(\begin{array}{cc}
Q_{M-1, M-1}^{(M-1)} & Q_{M-1, M}^{(M-1)} \\
Q_{M, M-1}^{(M-1)} & Q_{M, M}^{(M-1)}
\end{array}\right)=\left(f_{(M-1)}^{(M-1)}, f_{M}^{(M-1)}\right)
$$

- and finally, for $m=M$,

$$
A_{M}^{(M)} Q_{M, M}^{(M)}=f_{M}^{(M)} .
$$

In summary, the algorithm calculates the $Q^{(m)}$ matrix and $f^{(m)}$ array using the AGD software at each step (indexed by $m$ ) and solves each linear system induced by them at the Julia layer level in order to get the $A_{n}^{(m)}$ coefficients. Then, the $B_{n}^{(m)}$ coefficients are easily calculated from Equation (11).

\section{Numerical verifications}

\subsection{Limit cases in the far-field zone}

Algorithm verification was conducted via evaluation of the exact solution for $\left|f_{\infty}\right|$, obtained through the implemented codes for a fluid prolate (oblate) spheroidal scatterer, against known results valid in some limit cases (such as spheroid tending to a sphere, nonpenetrable Dirichlet and Neumann boundary conditions at the medium-spheroid interface).

A fluid spheroid approaching a sphere, with major semi-axis $a=1$ and minor semi-axis $b=0.99$, is assumed in order to compare the results predicted by the implemented codes with the classical solution for plane wave acoustic scattering by a fluid sphere [22]. Two different incident directions are considered, given by the line that contains both foci of an oblate spheroid (i.e. $\hat{x}$ incidence) and of a prolate spheroid (i.e. $\hat{z}$ incidence), in agreement with the coordinate system shown in Figure 1. The sphere has a reference radius $a=1$. Calculations were made for $k_{0}=5, k_{1}=3, \rho_{1} / \rho_{0}=3$. Scattering patterns obtained with the implemented numerical codes for fluid prolate/oblate spheroids fits the patterns due to spheres shown in Figure 2, Furthermore, tests were made to verify that the exact solution implemented for a fluid spheroids tends to the known results for impenetrable spheroids, modelling the latter scattererss through extreme density ratios in the former ones. For soft spheroid (or what is equivalent, Dirichlet case) [4, 5, 15, a density ratio $\rho_{1} / \rho_{0}=1 / 1000$ was assumed, whereas $\rho_{1} / \rho_{0}=1000$ was used to evaluate the rigid spheroid (equivalent to Neumann case) [1, 15. Computations were made for semi-axis values $a=1, b=0.25$ and for an arbitrary incidence angle, $\theta_{i}=2 \pi / 3$. Results are exhibited in Figures 3 and 4 . It can be observed there that in the limit of low density contrast, results for both spheroids tend to reproduce the Dirichlet case (Figure 3) while in the limit of high density contrast, they tend to reproduce Neumann case (Figure 4). Moreover, to illustrate intermediate scatterers' types, patterns for moderate density contrasts $\left(\rho_{1} / \rho_{0}=3\right)$ are shown.

\subsection{Results for the near-field zone}

Computation of both, $A_{m n}$ and $B_{m n}$ coefficients is necessary to obtain the scattered and transmitted acoustic pressures $p_{s}(\vec{r})$ and $p_{t}(\vec{r})$ according to Equations (2) and (3).

The total acoustic pressure at a field-point $\vec{r}$ is expressed as

$$
p_{\text {total }}(\vec{r})=\left\{\begin{array}{ccc}
p_{t}(\vec{r}) & \text { if } & \xi(\vec{r}) \leq \xi_{0} \\
p_{i}(\vec{r})+p_{s}(\vec{r}) & \text { if } & \xi(\vec{r})>\xi_{0}
\end{array}\right.
$$




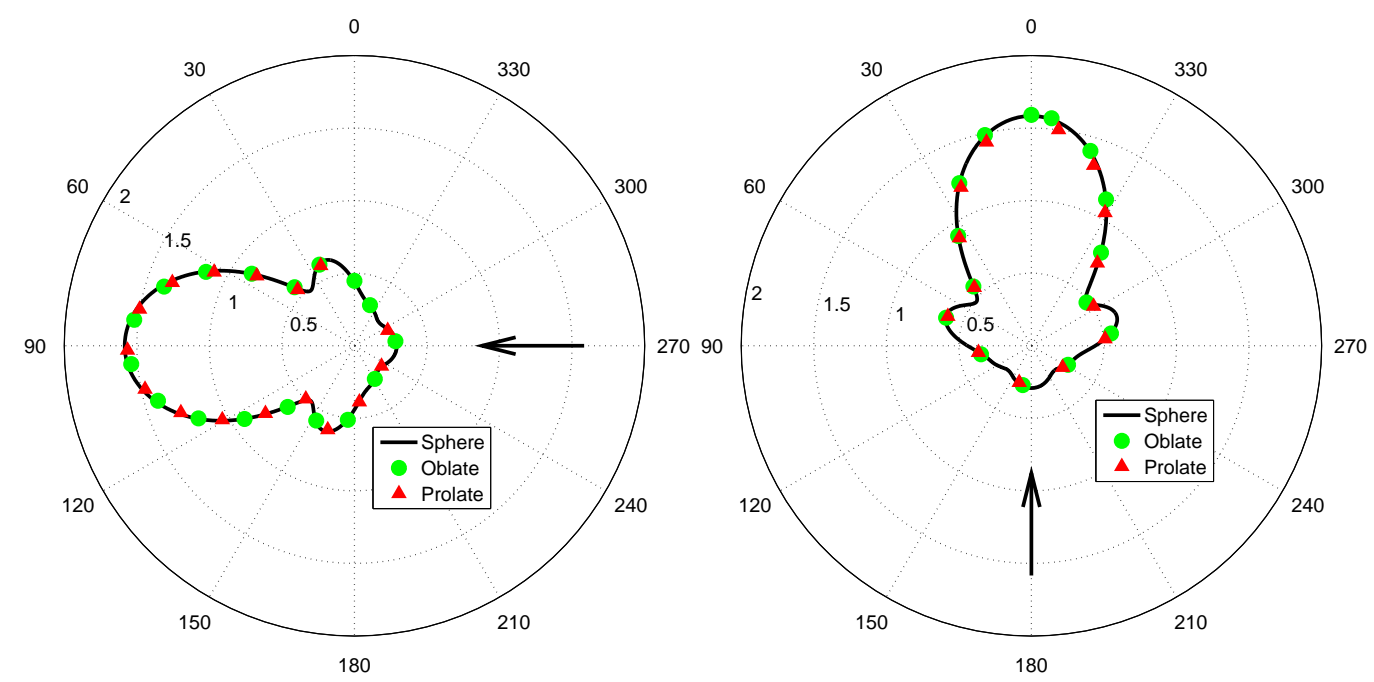

Figure 2: The absolute value of the oblate and prolate far-field scattering amplitude function $\left|f_{\infty}\right|$ tends to the absolute value of the sphere far-field scattering amplitude function when $b \rightarrow a$. The $\hat{x}$ incidence (left) and the $\hat{z}$ incidence (right) are shown.

Calculations are held for a prolate spheroid with semiaxes $a=2, b=1$, density ratio $\rho_{1} / \rho_{0}=1.5$ and wave numbers $k_{1}=1, k_{0}=1.5$. Results for the real part of the incident acoustic pressure $p_{i}$ and the absolute value of total and scattered acoustic pressures are shown in Figure 5 for the Cartesian planes $z=0$ and $y=0$. Computed total acoustic pressure verifies the continuity required by the boundary conditions at the interface $\xi=\xi_{0}$ as it is visually illustrated in Figure $5(b)$ and $(c)$.

\subsection{Comparison with reported results}

Among the relatively great number of publications addressing the acoustic scattering by penetrable spheroids, such as fluid spheroids immersed in a fluid surrounding medium, there is an article [11] that reports an analytical solution of the scattered pressure field for $\rho_{1} / \rho_{0} \not \approx 1, k_{1} / k_{0} \not \approx 1$ and small values of eccentricity $e$ (i.e. $\left.e=d /(2 a) \ll 1\right)$. In that paper a shape perturbation method is used to express the scattered pressure in terms of spherical wave functions, instead of spheroidal ones. Hence, approximate expressions are presented for any value of $e$ as a product of the scattering pressure by a sphere and a polynomial in even powers of $e$ till $\mathcal{O}\left(e^{6}\right)$. Moreover, in that reference some calculated expansion coefficients are presented in a Table for $\rho_{1} / \rho_{0}=1.22, k_{0} / k_{1}=1.27$ and $a / \lambda_{0}=0.9$, being $\lambda_{0}$ the acoustic wavelength in the medium. Those computed values lead to forward and backscattering cross-sections, $\sigma_{f}$ and $\sigma_{b}$, plotted in Figures 5 and 6 of the above mentioned work that can be compared with the corresponding results of the exact solution for $\left|f_{\infty}\right|$, obtained through the codes implemented here for a penetrable prolate spheroidal scatterer, given the relationships

$$
\begin{aligned}
\sigma_{f} & =4 \pi\left|f_{\infty}\left(\theta_{s}=\theta_{i}, \varphi_{s}=\varphi_{i}\right)\right|^{2} \\
\sigma_{b} & =4 \pi\left|f_{\infty}\left(\theta_{s}=\pi-\theta_{i}, \varphi_{s}=\varphi_{i}+\pi\right)\right|^{2}
\end{aligned}
$$

in far-field conditions.

Comparison between normalized backscattering and forward scattering cross-sections (i.e. $\left.\sigma / \lambda_{0}^{2}\right)$ predicted by the approximate solution [11] and the exact one computed through the implementation presented here is shown in Figure 6. Good agreement is achieved for 

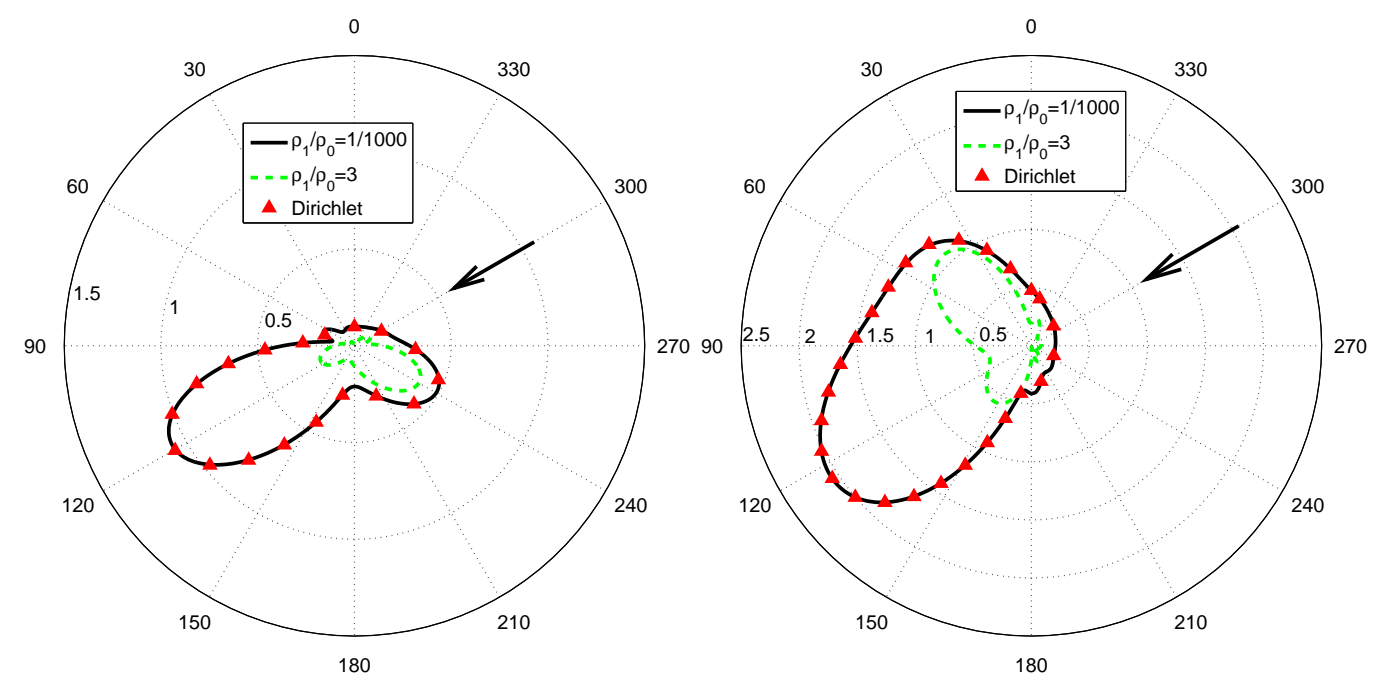

Figure 3: The form function $\left|f_{\infty}\right|$ with two cases of the density ratio and the Dirichlet case. Prolate (left) and oblate (right) cases.

the forward scattering case while the agreement gets worse for increasing eccentricity when backscattering is considered. For $e=0.4$ (i.e. $b / a>0.9$, which is quite close to be a spherical shape), $\sigma_{b}$ takes undesirable negative values at incident angles greater than $54^{\circ}$, which has no physical sense whereas the exact solution predicts positive values as expected.

\section{$5 \quad$ Numerical results}

\subsection{Applications to aquatic ecosystem research}

In acoustical oceanography, the Prolate Spheroidal Model (PSM) [1, 4, 5], provides a useful tool for determining target strength $(T S)$ of individual fish and volume scattering strength $\left(S_{V}\right)$ of zoo and phytoplankton. In this research field, it is useful to estimate their numerical abundance as well as to improve morphological and anatomical representations of the volume scatterers present at sea.

In fisheries acoustics, as well as in other SONAR applications, sound scattering by an object is analyzed in the logarithmic scale using the target strength parameter, $T S$, that can be expressed as

$$
T S_{b s}(\text { re. } 1 \mathrm{~m})=20 \log \left(\left|f_{\infty}\left(\theta_{s}=\pi-\theta_{i}, \varphi_{s}=\varphi_{i}+\pi\right)\right|\right),
$$

Equation (12), has length dimensions. where $\left|f_{\infty}\right|$ has length dimensions. In particular, for the prolate spheroidal scatterer, $f_{\infty}$ is defined in Equation (12).

Previously, other investigators using the PSM have reported solutions on scattering by fluid spheroids for some cases of interest such as the so-called weakly scattering (i.e. $c_{1} / c_{0} \approx 1$ and $\rho_{1} / \rho_{0} \approx 1$ ) and the gas-filled case [23]. More precisely, Furusawa [4] worked with $1.01 \leq \rho_{1} / \rho_{0} \leq 1.07$. Other authors limited their calculations to certain range of frequencies [6, 19].

\subsection{Weakly-Scattering by a prolate fluid spheroid}

When the medium and the prolate spheroid have similar physical properties, i.e. $\rho_{1} / \rho_{0} \approx 1$ and $c_{1} / c_{0} \approx 1$, a usual approximation consists in assuming that the non-diagonal matrix 

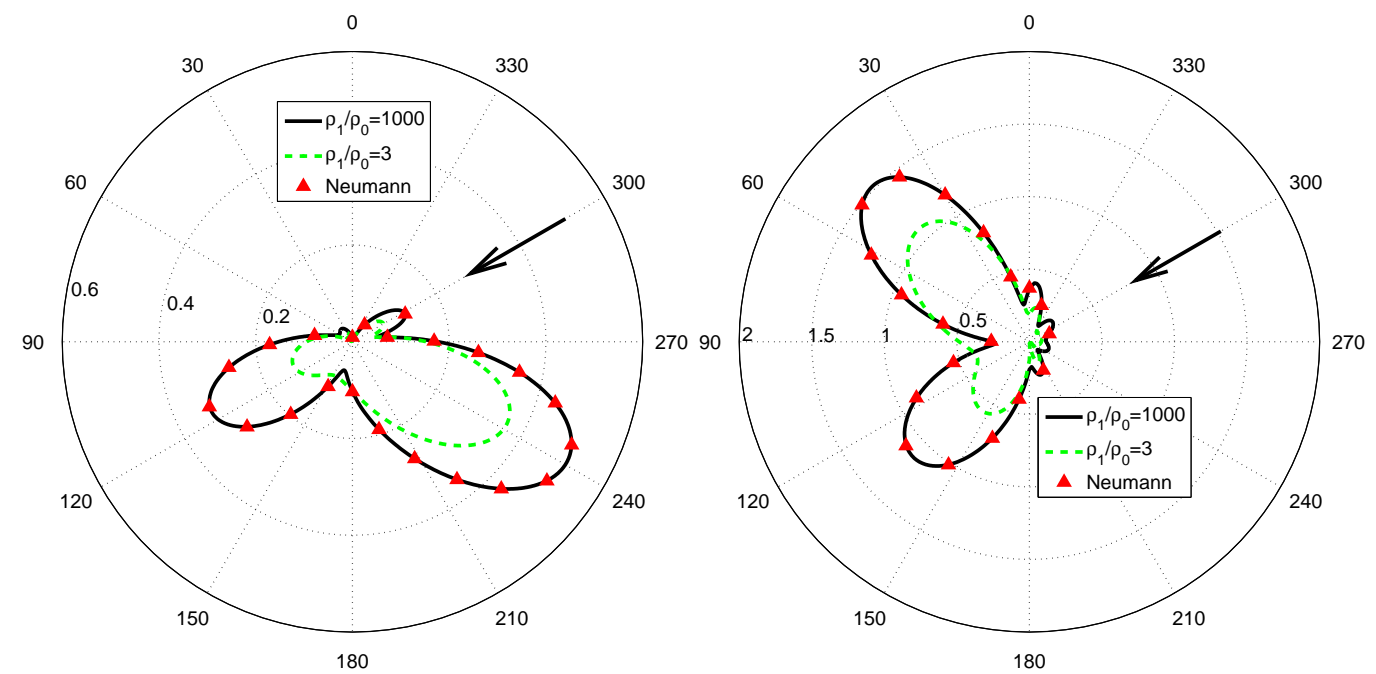

Figure 4: The form function $\left|f_{\infty}\right|$ with two cases of the density ratio and the Neumann case. Prolate (left) and oblate (right) cases.

elements $\alpha_{n \ell}^{(m)}$ defined in Equation (7) can be neglected [4, 5, 6], thus

$$
\alpha_{n \ell}^{(m)} \approx \alpha_{n n}^{(m)} \delta_{n \ell}
$$

whereas the matrix system given by Equation 13 becomes almost trivial so that straightforward calculations lead to the approximate coefficients,

$$
A_{m n}^{\text {approx }}=-\frac{E_{n}^{m(1)}}{E_{n}^{m(3)}}
$$

where

$$
E_{n}^{m(i)}=R_{m n}^{(i)}\left(h_{0}, \xi_{0}\right)-\frac{\rho_{1} R_{m n}^{(1)}\left(h_{1}, \xi_{0}\right)}{\rho_{0} R_{m n}^{(1)^{\prime}}\left(h_{1}, \xi_{0}\right)} R_{m n}^{(i)^{\prime}}\left(h_{0}, \xi_{0}\right) .
$$

It is worthy to note that the approximation indicated in Equation 14 leads to an explicit expression for the $A_{m n}$ coefficients as set in Equation (15). This crucial approximation, which avoids the numerically heavy task of solving the successive matrix systems, is very often used and it turns out to be appropriate in several cases of interest such as weakly scattering, low eccentricity and low frequencies. However, there are cases of interest where this approximation can not be used. On the other hand, solving the exact case through the matrix system is somewhat tricky because the matrix $Q_{\ell j}^{(m)}$ may be illconditioned when $\left|c_{1} / c_{0}-1\right| \gg 1$. To avoid errors emerging from the numerical solution of the matrix system, algebra of precision beyond hardware floating point (64 bits) has been employed.

In order to compare results in the case of weakly scattering, the value of TS derived from the exact coefficients' computation through the solution (Equation (13)) with the one derived from the approximate solution (Equation (15)), a prolate spheroid insonified at 38 $\mathrm{kHz}$, has been considered. The input data have been $\rho_{1}=1028.9 \mathrm{~kg} \mathrm{~m}^{-3}$ and $c_{1}=1480.3$ $\mathrm{m} \mathrm{s}^{-1}, a=0.1 \mathrm{~m}, b=0.01 \mathrm{~m}$ for the spheroid immersed in seawater characterised by $\rho_{0}=1026.8 \mathrm{~kg} \mathrm{~m}^{-3}$ and $c_{0}=1477.4 \mathrm{~m} \mathrm{~s}^{-1}$.

Comparison between both solutions is shown in Figure 7(A) for the whole $0^{\circ}$ to $90^{\circ}$ incidence angle-range. It can be observed that both curves do not fit. Furthermore, 
it should be noted that the used approximation (Equation (14p) to compute the matrix elements $Q$, defined in Equation (8), leads to detect nearly no differences between the exact and the approximate solutions for TS, whereas using this approximation to compute the vector components $f$ (Equation (9)), generates very significant differences when the density contrast verifies $\rho_{1} / \rho_{0} \lesssim 1.002$ like in this case. However, if the sound speed contrast is $c_{1} / c_{0} \approx 1$ but the density contrast is for example $\rho_{1} / \rho_{0}=1.06$, the differences between the approximate and exact solutions is roughly $0.6 \mathrm{~dB}$.

Additionally, in the frame of the approximate solution to compute TS (Equation (15)), the maximum frequency range previously reported [6] could be extended till $h_{0}=762.08$. Results are shown in Figure 7(B) for $25 \mathrm{kHz} \leq \mathrm{f} \leq 1800 \mathrm{kHz}$ and $\theta_{i}=90^{\circ}$, where the series in Equation (2) was truncated at $m \leq M=150$ and $n \leq M+m$, so that a total of 22801 modes were summed. A previously published algorithm suitable for high values of $h$ was used in order to compute prolate spheroidal wave functions [24].

\subsection{Gas-Filled Prolate spheroid}

A gas-filled spheroid is a fluid spheroid with density and sound speed contrasts similar to the ones corresponding to the water-air interface (i.e. $c_{1} / c_{0}<1$ and $\rho_{1} / \rho_{0} \ll 1$ ). This case has a wide spectrum of applications in acoustical oceanography. In particular, at intermediate frequencies, the fish swimbladder may be considered as a gas-filled or a soft-scatterer when the PSM is used.

At low frequency $\left(k_{0} a \ll 1\right)$, it is possible to use an approximation similar to Equation (14) instead of solving the exact Equation (13) [7]. Scattering by a gas-filled prolate spheroid for $k_{0} a \ll 1$ has already been theoretically investigated under the approach of the PSM, and some numerical results were published [23]. However, at intermediate and high frequencies, the gas-filled prolate spheroid has not been modelled with the PSM [6, 19], hence, other approximate alternatives were used. More precisely, Jech et al. [6] reported that convergence was not achieved for the following input data: $\rho_{0}=1026.8 \mathrm{~kg} \mathrm{~m}^{-3}$, $\rho_{1}=1.24 \mathrm{~kg} \mathrm{~m}{ }^{-3}, c_{0}=1477.4 \mathrm{~m} \mathrm{~s}^{-1}, c_{1}=345.0 \mathrm{~m} \mathrm{~s}^{-1}, a=0.07 \mathrm{~m}, b=0.01 \mathrm{~m}$ and $f=38 \mathrm{kHz}$. Then, in that work, TS values from a gas-filled spheroid were computed through different methods such as the Boundary Element Method and Kirchhoff ray mode. The same spheroid has been considered in this work, and convergence was achieved. Results are shown in Figure 8, where it can be seen that the soft-scatterer and the exact solution have the same behaviour. Furthermore, it can be observed that the approximate solution given by Equation (15) clearly cannot be used in this case.

\section{Routines' sintaxis}

The provided routines for far-field patterns computation when plane acoustic waves incide on prolate or oblate spheroids were developed in Julia programming language, which has a MATLAB-like sintaxis, so that MATLAB and Octave users are expected to get easily familiarized with it. They can be freely downloaded from a GitHub repository https://github.com/elavia/liquid_spheroid as a ZIP file or as individual files and they can be directly executed from the Julia REPL interface.

The material uploaded in the repository is organized in two folders, namely, Linux and Windows, since the routines have been implemented for both operative systems. They were developed and tested in Julia 0.5 under Fedora Linux and in Julia 0.4 under Windows 7, respectively. For ensuring their correct functionality, previous installation of Julia's GSL 
package (Julia implementation of the GNU Scientific Library) is required ${ }^{1}$

The files JUL.routines. $j 1$ and JUL . auxiliar. $j 1$ contain the main and auxiliary functions for the expansion coefficients and the far-field patterns calculations. Several Julia scripts reproducing the results presented in Section 4.1 are also provided. Their names were chosen so that they are related to their particular purpose. Hence, it is not a surprise for example that script.oblate_liquid.jl provides the pattern for an oblate spheroid in the fluid case.

The AGD executable files obl_sphwv and pro_sphwv constitute the numerical engine for the calculation of the spheroidal wave functions inside Julia routines and they are called through specialized shell scripts (.sh files, under Linux) and batch files (.bat files, under Windows). Those executable files were taken from the scattering-master/spheroidal/sphwv directory in the AGD software source tree and they can be downloaded from the authors' site at https://github.com/radelman/scattering.

For a detailed description of the AGD software and the subtleties in the calculation of spheroidal wave functions, the reader is referred to the original reference that partially inspired this work [15]. The parameters configuration for the AGD software execution inside the Julia routines has been done trough the text files obl.parameters and pro.parameters. In these files safe values of the parameters were taken in order to ensure reliable results. It is remarkable that input selection associated with extreme cases, such as high frequency, may lead to an unpredicted behaviour, whose comprehension requires deeper understanding of the AGD code. However, these cases are far beyond the scope of the provided routines.

The first step to begin working with the code, once the Julia environment is properly installed, is to download the routines from the authors' repository, decompress them in a directory and start the Julia REPL interface from that directory. Then, main and auxiliary functions must be loaded at the Julia's prompt through the script JUL.main.jl, before any other script is run. Afterwards all the routines are available in the current workspace and can be called from the prompt.

In order to illustrate and clarify the routines' operation, an example session is presented. First, the routines are loaded through the inclusion of the main script, i.e.

julia > include("JUL.main.jl") ;

Therefore, the routines are in the workspace and it is now possible, for example, to calculate the far-field pattern $\left|f_{\infty}\right|$ in the fluid case for a prolate spheroid. This is accomplished with the command

julia > include("script.prolate_liquid.jl") ;

The pattern is saved in a file called Out.Pattern. dat within the current directory. The data format in this file is: angle (in radians) and absolute value of the $f_{\infty}$ (in arbitrary length units compatible with the ones used for $a, b, k)$. The computing elapsed time is strongly dependent on the computer's speed and the parameters set in the AGD software. All the provided scripts save the results in the same file so that the content is overwritten each time. Scripts output can be plotted with any plotting software (In particular, the plots in this work were made using MATLAB and Gnuplot).

The scripts have a section of user-defined parameters. In the above mentioned example, script.prolate_liquid.jl contains

\footnotetext{
${ }^{1}$ In most cases it is enough to type Pkg. add (“GSL") at the Julia's prompt.
} 


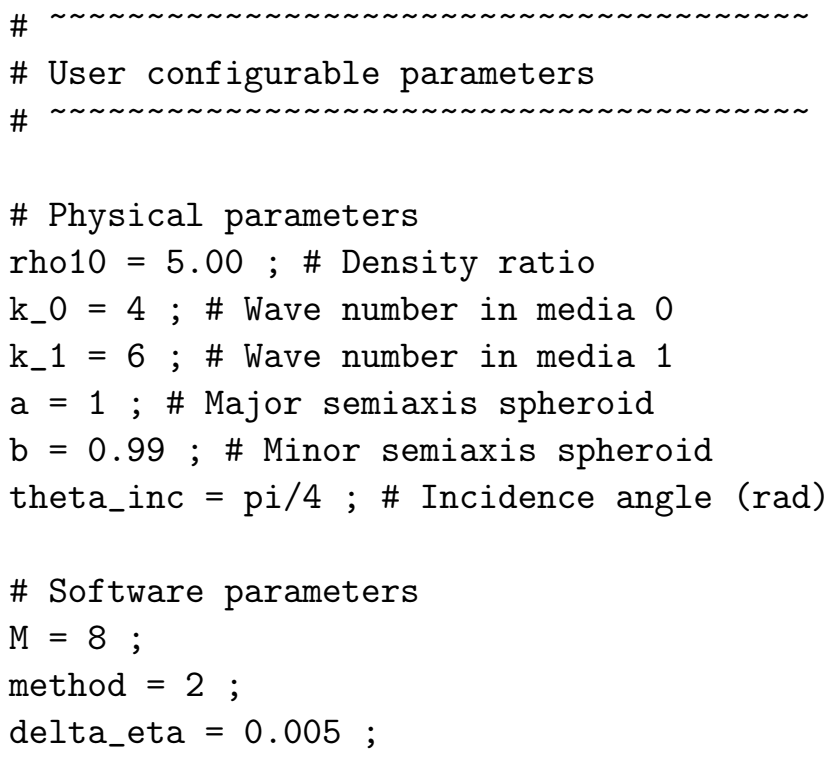

The meaning of the physical parameters, namely, $\rho_{10}, k_{0}, k_{1}, a, b, \theta_{i}$ is evident from the explicative text after the commentary character \#. The software parameters section includes parameters related to the spheroidal wave calculation. The M parameter is the maximum value taken by $m$ in the coefficients $A_{m n}, B_{m n}$ and is directly associated to the total number of the coefficients to be calculated. For instance, $M=8$ value means a total of $(M+1)(M+2) / 2=45$ coefficients. The method parameter refers to the type of calculation (method $=1$ is convenient for $\xi \gg 1$ and method $=2$ for $\xi \sim 1$ ). Finally, delta_eta is the step on the $\eta$-grid and as a consequence of the inequality $-1 \leq \eta \leq 1$, that value leads to a $2 /$ delta_eta grid size.

\section{Discussion and conclusions}

A novel implementation of the exact analytical solution for the problem of acoustic scattering by fluid or penetrable prolate/oblate spheroids governed by Helmholtz equation, is presented. A set of computational routines to calculate the expansion coefficients appearing in the mathematical expressions of the scattered and transmitted acoustic pressures is provided. These routines are freely available from the authors' GitHub repository. The coefficients' computation is precisely the most cumbersome task related to numerical calculation of the mathematical expressions above mentioned.

The results predicted by the computational implementation were tested for the geometrical limit when both prolate/oblate spheroids tend to the sphere and for the physical limit when they tend to soft or rigid spheroidal scatterers, taking low and high values of dentisities ratios, respectively. In the latter two cases predicted results turn out to be in agreeement with expected ones for Dirichlet and Neumann boundary conditions. Additionally, it has been qualitatively verified, trough the calculation for near-field conditions, that the numerical solution satisfies the boundary condition at the surface of the spheroids. The implemented exact solution has been successfully compared against a previously reported approximate solution and extended to eccentricity ranges where the approximation does not work properly.

Furthermore, the implemented solution enabled to extend the applicability ranges previously published in the literature for cases of interest in aquatic ecosystem research. Table 1 summarizes the physical properties, the geometry and type of spheroid and the acoustic frequency range of the penetrable scatterer considered by different authors. 


\begin{tabular}{|c|c|c|c|c|c|}
\hline Reference & $\rho_{1} / \rho_{0}$ & $c_{1} / c_{0}$ & $h_{0}(\max )$ & $b / a(\min )$ & $\begin{array}{c}\text { Type of } \\
\text { Spheroid }\end{array}$ \\
\hline Yeh, 1967 8] & 0.50000 & 1.000 & 9.38 & 0.109 & Prolate \\
\hline Furusawa, 1988 [4] & 1.07000 & 1.050 & 12.00 & 0.100 & Prolate \\
\hline Ye et al., 1998 [23] & 0.00129 & 0.220 & 0.73 & 0.050 & $\begin{array}{c}\text { Prolate } \\
\text { (low freq.) }\end{array}$ \\
\hline Okumura et al., 2003 [19] & 1.05000 & 1.050 & 9.79 & 0.200 & Prolate \\
\hline Tang et al.,2009 [10] & 1.04000 & 1.020 & 24.84 & 0.150 & Prolate \\
\hline Prario et al., 2015 [5] & 1.06800 & 1.088 & 120.00 & 0.238 & Prolate \\
\hline Jech et al., 2015 [6] & 1.00200 & 1.002 & 118.00 & 0.143 & Prolate \\
\hline This work & $\begin{array}{l}3.00000 \\
0.00100 \\
1000.00000 \\
0.00129\end{array}$ & $\begin{array}{l}0.600 \\
0.220 \\
1.500 \\
1.270\end{array}$ & 762.08 & 0.100 & $\begin{array}{l}\text { Prolate/ } \\
\text { Oblate }\end{array}$ \\
\hline
\end{tabular}

Table 1: Physical properties, the geometry and type of spheroid and the acoustic frequency range of the penetrable scatterer considered by different authors (i.e. density and sound speed contrasts; $h_{0}$ maximum, $b / a$ ratio and prolate and/or oblate spheroid). 
With the aim of generating the computational codes presented in this work, the efficient software by AGD was used in addition to the numerical capabilities of the new scientific programming language Julia. Thus, it was possible to take advantage of the arbitrary floating point precision support in both pieces of code. Moreover, scripts to reproduce the calculations presented in Section 4.1 are also provided to enable a minimum consistency check.

The developed codes are released intending to contribute with researchers working in acoustic scattering in order to simplify the tedious algorithmic task involved in the resolution of the cumbersome matrix system when calculating the exact solution for a fluid spheroidal scatterer.

\section{Acknowledgements}

This work was supported by the PIDDEF Program of the Argentinian Ministry of Defense (13-2014), the Argentinian Navy and the National Council for Scientific and Technical Research (CONICET).

The authors wish to remark once more the outstanding piece of software that Adelman and his collaborators have made freely available to the scientific community. It was a real pleasure to work with it. Of course, the same applies to Julia programming language. Moreover, they acknowledge the work of the programmers' team that have designed and implemented that language. It is marvelous. With both they are in debt. 


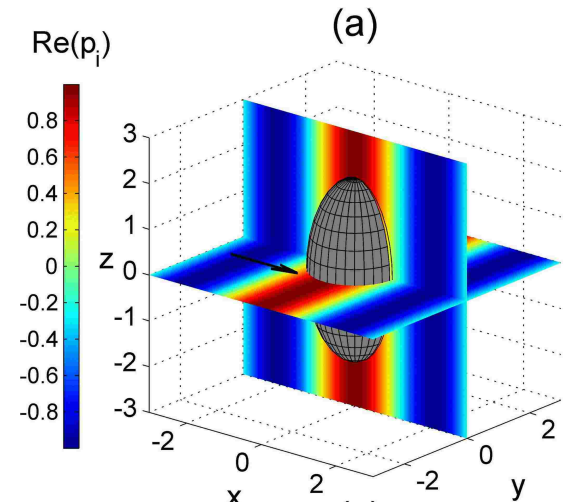

(c)

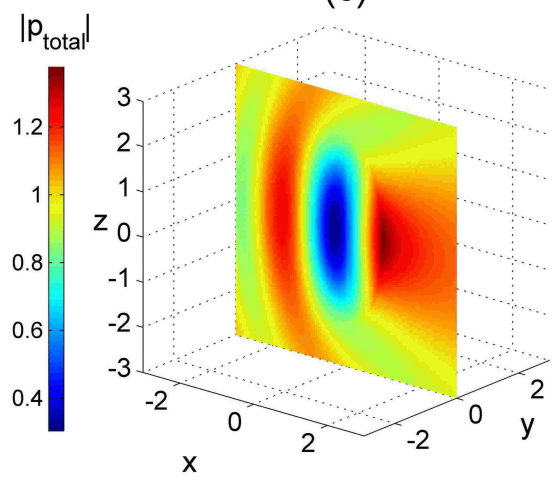

(b)

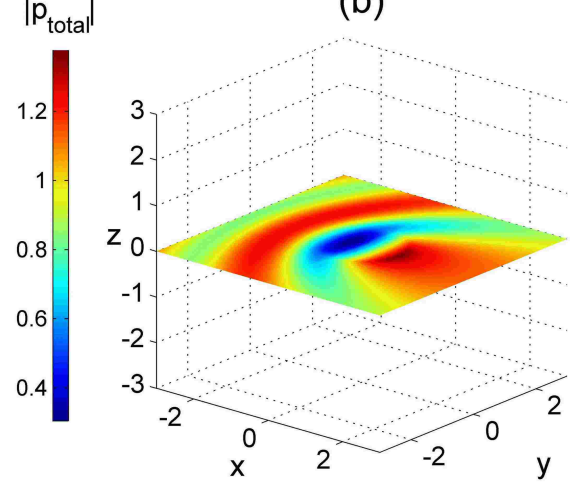

(d)
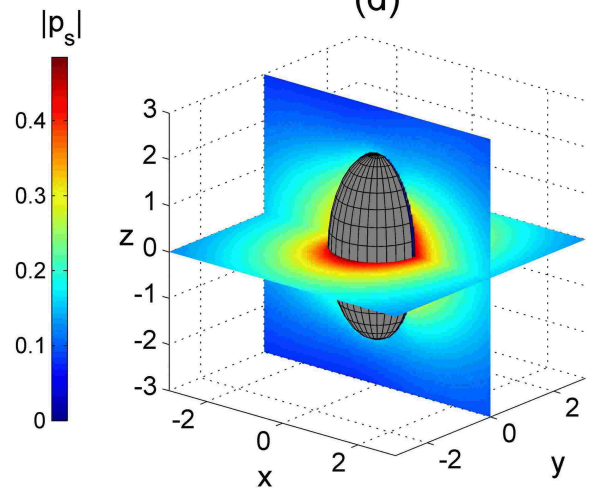

Figure 5: (a) Real part of incident acoustic pressure, $\operatorname{Re}\left(p_{i}\right)$, at the Cartesian planes $z=0$ and $y=0$. (b) Absolute value of total acoustic pressure, $\left|p_{\text {total }}\right|$, at $z=0$. (c) Absolute value of total acoustic pressure, $\left|p_{\text {total }}\right|$, at $y=0$. (d) Absolute value of scattered acoustic pressure, $\left|p_{s}\right|$, at $z=0$ and $y=0$. Assumed values: $a=2, b=1$, density ratio $\rho_{1} / \rho_{0}=1.5$, wave numbers $k_{1}=1, k_{0}=1.5$ and incidence angle $\theta_{i}=\pi / 2$. 

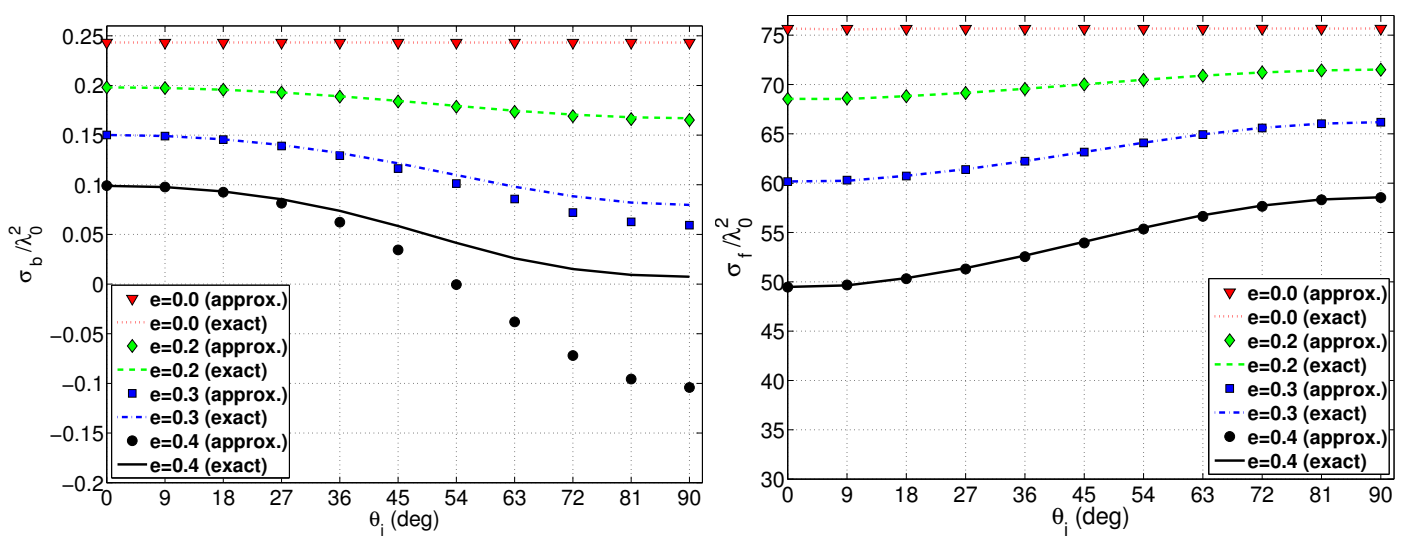

Figure 6: Comparison between exact solution and analytical approximation previously published for prolate spheroids with different kinds of excentricity e. Normalized backscattering cross section (left) and forward-scattering cross section (right). Both are normalized by $\lambda_{0}$, the wavelength in the medium.

(A)

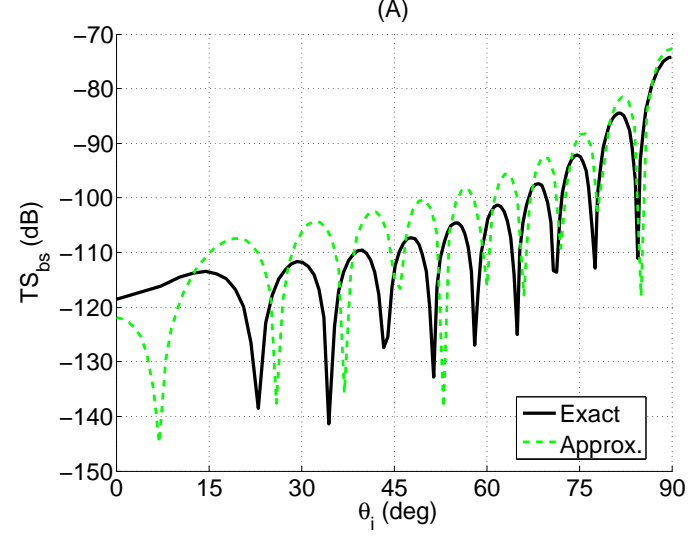

(B)

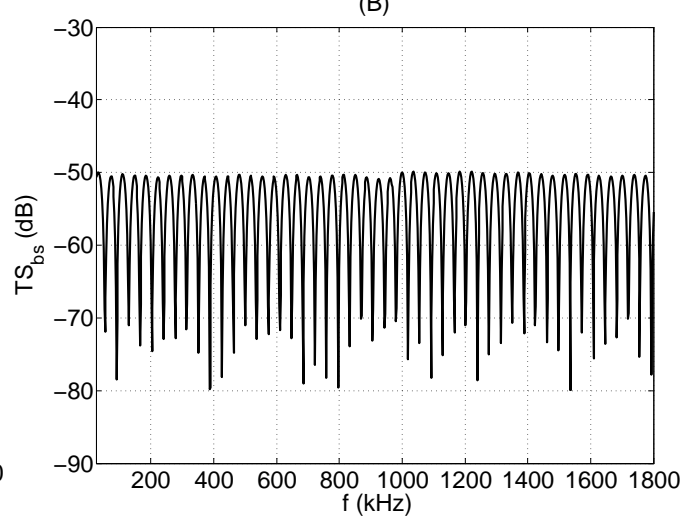

Figure 7: (A) TS vs. incident angle, comparison between exact and approximate solutions for a prolate spheroid. $\rho_{0}=1026.8 \mathrm{~kg} \mathrm{~m}^{-3}, \rho_{1}=1028.9 \mathrm{~kg} \mathrm{~m}^{-3}, c_{0}=1477.4 \mathrm{~m} \mathrm{~s}^{-1}$, $c_{1}=1480.3 \mathrm{~m} \mathrm{~s}^{-1}, a=0.1 \mathrm{~m}, b=0.01 \mathrm{~m}, f=38 \mathrm{kHz}$. (B) TS $v s$. frequency at $\theta_{i}=90^{\circ}$ for a spheroid with the same parameters than (A) but $\rho_{1}=1088.4 \mathrm{~kg} \mathrm{~m}^{-3}$. 


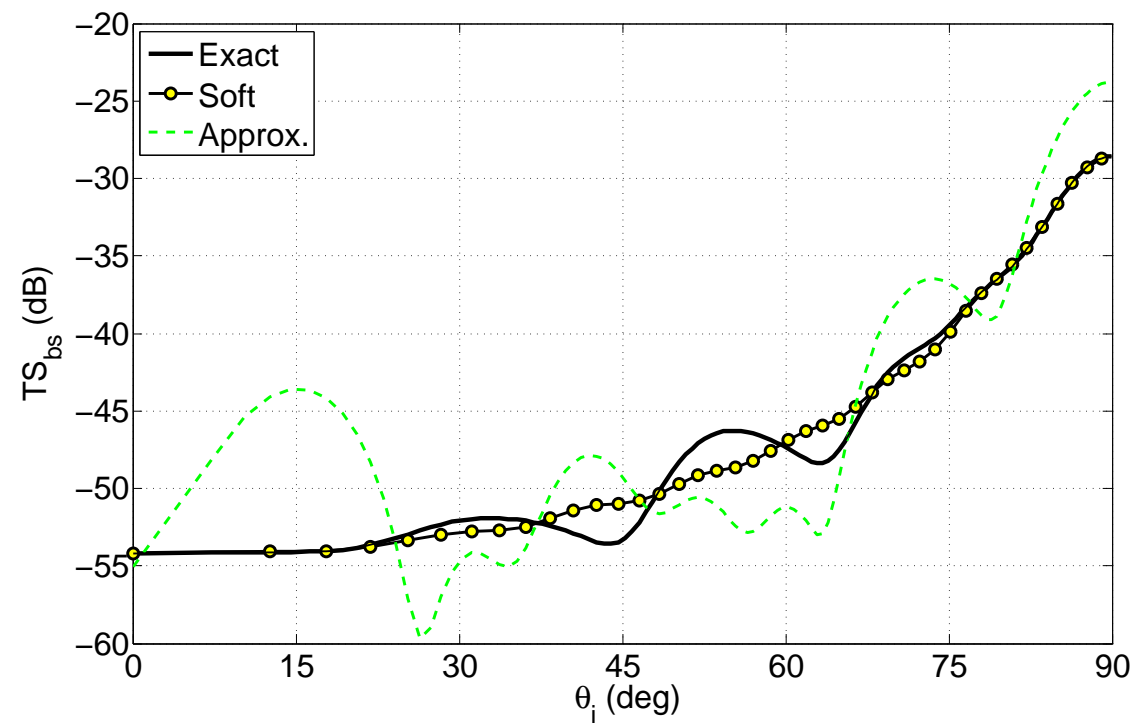

Figure 8: TS vs. incident angle for a gas-filled. $\rho_{0}=1026.8 \mathrm{~kg} \mathrm{~m}^{-3}, \rho_{1}=1.24 \mathrm{~kg} \mathrm{~m}^{-3}, c_{0}=1477.4$ $\mathrm{m} \mathrm{s}^{-1}, c_{1}=345.0 \mathrm{~m} \mathrm{~s}^{-1}, a=0.07 \mathrm{~m}, b=0.01 \mathrm{~m}$ and $\mathrm{f}=38 \mathrm{kHz}$. 


\section{References}

[1] R. Spence, S. Granger: The scattering of sound from a prolate spheroid. The Journal of the Acoustical Society of America 23 (1951) 701-706.

[2] A. Silbiger: Scattering of sound by an elastic prolate spheroid. The Journal of the Acoustical Society of America 35 (1963) 564-570.

[3] D. S. Burnett: Radiation boundary conditions for the helmholtz equation for ellipsoidal, prolate spheroidal, oblate spheroidal and spherical domain boundaries. Journal of Computational Acoustics 20 (2012) 1230001.

[4] M. Furusawa: Prolate spheroidal models for predicting general trends of fish target strength. Journal of the Acoustical Society of Japan (E) 9 (1988) 13-24.

[5] I. Prario, J. Gonzalez, A. Madirolas, S. Blanc: A prolate spheroidal approach for fish target strength estimation: Modeling and measurements. Acta Acustica united with Acustica 101 (2015) 928-940.

[6] J. M. Jech, J. K. Horne, D. Chu, D. A. Demer, D. T. Francis, N. Gorska, B. Jones, A. C. Lavery, T. K. Stanton, G. J. Macaulay et al.: Comparisons among ten models of acoustic backscattering used in aquatic ecosystem research. The Journal of the Acoustical Society of America 138 (2015) 3742-3764.

[7] C. Yeh: The diffraction of sound waves by penetrable disks. Annalen der Physik 468 (1964) 53-61.

[8] C. Yeh: Scattering of acoustic waves by a penetrable prolate spheroid. i. liquid prolate spheroid. The Journal of the Acoustical Society of America 42 (1967) 518-521.

[9] N. Gorska, D. Chu: Some aspects of sound extinction by zooplankton. The Journal of the Acoustical Society of America 110 (2001) 2315-2325.

[10] Y. Tang, Y. Nishimori, M. Furusawa: The average three-dimensional target strength of fish by spheroid model for sonar surveys. ICES Journal of Marine Science: Journal du Conseil 66 (2009) 1176-1183.

[11] A. Kotsis, J. Roumeliotis: Acoustic scattering by a penetrable spheroid. Acoustical Physics 54 (2008) 153-167.

[12] V. C. Anderson: Sound scattering from a fluid sphere. The Journal of the Acoustical Society of America 22 (1950) 426-431.

[13] P. M. Morse, H. Feshbach: Methods of theoretical physics. McGraw Hill Book Company, New York, 1953.

[14] E. Skudrzyk: The foundations of acoustics: basic mathematics and basic acoustics. Springer-Verlag, Wien, 1971.

[15] R. Adelman, N. A. Gumerov, R. Duraiswami: Software for computing the spheroidal wave functions using arbitrary precision arithmetic. arXiv:1408.0074v1 [cs.MS] (2014).

[16] R. Adelman, N. A. Gumerov, R. Duraiswami: Semi-analytical computation of acoustic scattering by spheroids and disks. The Journal of the Acoustical Society of America 136 (2014) EL405-EL410. 
[17] J. Bezanson, A. Edelman, S. Karpinski, V. B. Shah: Julia: A fresh approach to numerical computing. arXiv preprint arXiv:1411.1607 (2014).

[18] A. L. Van Buren, J. E. Boisvert: Accurate calculation of prolate spheroidal radial functions of the first kind and their first derivatives. Quarterly of Applied Mathematics 60 (2002) 589-599.

[19] T. Okumura, T. Masuya, Y. Takao, K. Sawada: Acoustic scattering by an arbitrarily shaped body: an application of the boundary-element method. ICES Journal of Marine Science: Journal du Conseil 60 (2003) 563-570.

[20] A. N. Lowan: Spheroidal wave functions. - In: Handbook of Mathematical Functions. M. Abramovitz, I. A. Stegun (eds.). Dover Publications Inc. New York, 1972, 751-759.

[21] C. Flammer: Spheroidal wave functions. Standford University press, Standford, California, 1957.

[22] R. Hickling: Analysis of echoes from a solid elastic sphere in water. The Journal of the Acoustical Society of America 34 (1962) 1582-1592.

[23] Z. Ye, E. Hoskinson: Low-frequency acoustic scattering by gas-filled prolate spheroids in liquids. ii. comparison with the exact solution. The Journal of the Acoustical Society of America 103 (1998) 822-826.

[24] A. L. Van Buren: Mathieu and spheroidal wave functions fortran programs for their accurate calculation. http://www.mathieuandspheroidalwavefunctions.com, last viewed on 3 May 2016. 